\title{
Modification of Carbon Nanotubes with Fluorinated Ionic Liquid for Improving Processability of Fluoro-Ethylene-Propylene
}

\author{
Hongyang $\mathrm{Ma}^{1,2, *}$, Benjamin $\mathrm{Chu}^{1}$, and Benjamin S. Hsiao ${ }^{1, *}$ \\ ${ }^{1}$ Department of Chemistry, Stony Brook University, Stony Brook, NY11794-3400, USA \\ ${ }^{2}$ State Key Laboratory of Organic-Inorganic Composites, Beijing University of Chemical \\ Technology, Beijing 100029, China
}

\section{Corresponding authors}

Phone: (631)229-6899 (H.M.); (631)632-7793 (B.S.H); Fax: (631)632-6518

E-mail: mahy@mail.buct.edu.cn (H.M.); benjamin.hsiao@stonybrook.edu (B.S.H.) 
Abstract: Fluorinated ionic liquid (F-IL), 1-(3-perfluorooctylpropyl)-3-methylimidazolium bis(perfluoroethylsufonyl)amine, had been successfully prepared and employed to modify multi-wall carbon nanotubes (MWCNTs) for improving the processability of fluoro-ethylenepropylene (FEP). The thermally decomposed temperature of F-IL was higher than $350{ }^{\circ} \mathrm{C}$ measured by thermal gravimetric analysis (TGA) which indicated that the fluorinated ionic liquid could be suitable for melting blend with FEP (blending at $290{ }^{\circ} \mathrm{C}$ ) by a twin-screw extruder. Through "cation- $\pi$ " interaction between the imidazolium cation of F-IL and the graphene surface of MWCNTs, MWCNTs can be modified with F-IL and used as nanofillers to improve the dispersity of MWCNTs in fluorocopolymer FEP verified by SEM images of the FEP nanocomposite. The structural characterization and mechanical property of FEP nanocomposite during the deformation were investigated by tensile experiments and simultaneous time-resolved wide-angle X-ray diffraction (WAXD) and small-angle X-ray scattering (SAXS) techniques.

Keywords: fluorinated ionic liquid; MWCNTs; fluorocopolymer; mechanical property; processability 


\section{Introduction}

Typical fluoropolymers such as poly(tetrafluoroethylene) (PTFE), fluoro-ethylenepropylene (FEP) copolymer, and perfluoroalkoxy (PFA) copolymer, are recognized as the materials with extremely poor processability due to the strong interactions between polymer chains and the low surface energy, which definitely limited the applications of those polymers, although they exhibit excellent chemical resistance to acids, bases, and solvents, as well as good mechanical properties and high temperature stability [1-3]. On the other aspect, carbon nanotubes (e.g. MWCNTs) are nano-scale materials with super thermal, mechanical, and conductive properties [4], and they could be used as nanofillers to improve the mechanical and processable properties of fluoropolymer materials, therefore, the dispersion of carbon nanotubes into fluoropolymer matrix has attracted great interesting and attentions [5-7]. Due to the low surface energy and solvent inert property of fluoropolymers, surface modification of carbon nanotubes was regarded as an effective strategy and was employed to improve the dispersity of carbon nanotubes in the fluoropolymer matrix $[8,9]$. Direct fluorination of carbon nanotubes was considered as an effective approach which certainly improves the compatibility of carbon nanotubes in different fluoropolymer matrices [5]. Moreover, the surface grafting functional group (e.g. fluoroalkyl group) onto carbon nanotubes can also enforce the compatibility between nanotubes and fluoropolymers [7]. After chemical modifications, however, the thermal stability of carbon nanotubes was much decreased and two more reactions have to be involved in the modification process. Therefore, new approaches other than chemical modifications should be explored to address this problem.

Single-walled carbon nanotubes (SWCNTs) could form a gel with ionic liquids based on "cation- $\pi$ " interaction between the surface of SWCNTs and the imidazolium cation of the ionic liquid, which can dramatically improve the mechanical property and electrical 
conductivity of their nanocomposite materials [10-13]. Furthermore, tri-alkyl imidazolium salts and MWCNTs were melt-extruded into polystyrene and a better dispersion of MWCNTs in polystyrene was observed with the addition of ionic liquids [14]. By using X-ray diffraction (XRD) and infrared (IR), they proposed a physical interaction between MWCNTs and the long aliphatic chain of an ionic liquid. Recently, ionic liquids with long alkyl substituted groups were employed to modify carbon nanofibers through physical "cation- $\pi$ " interaction between the imidazolium cation of the ionic liquid and the para-arene surface of carbon nanofibers, where the long alkyl substituted group of the ionic liquid improved the compatibility of carbon nanofibers in ultra-high molecular weight polyethylene (UHMWPE) [15-19]. As a result, the toughness of UHMWPE has been essentially improved due to the possible mechanism of sliding between UHMWPE crystal regions and the surface of carbon nanofibers during the tensile deformation.

Encouraging by this discovery, a fluorinated ionic liquid had been prepared and employed to modify the surface of MWCNTs, where the fluoro-substituted group of the cationic part of the ionic liquid could improve the dispersity of MWCNTs in fluoropolymer matrices. The fluorocopolymer nanocomposites prepared with FEP and F-IL-MWCNTs by melting extrusion exhibited improved mechanically tensile properties, and the structural changes during the deformation of the nanocomposites were monitored with in-situ WAXD and SAXS technologies.

\section{Experimental}

\subsection{Materials}

Fluoro-ethylene-propylene (Teflon FEP 100) pellets were provided by DuPont, USA. The multi-wall carbon nanotubes (MWCNTs) were obtained from the Nanostructured and Amorphous Materials, Inc., where the diameter of MWCNTs was about 8-15 nm, and the 
purity was greater than $95 \%$ 3-(perfluorooctyl)propyl iodide, 1-methylimidazole, and other reagents were purchased from Aldrich, and so the bis(perfluoroethylsulfonyl)amide lithium salts $(\mathrm{FC}-130,3 \mathrm{M})$ were used as received.

\subsection{Synthesis of Fluorinated Ionic Liquid}

The ionic liquid F-IL was synthesized by following the procedures as shown in Scheme 1 [20-22].

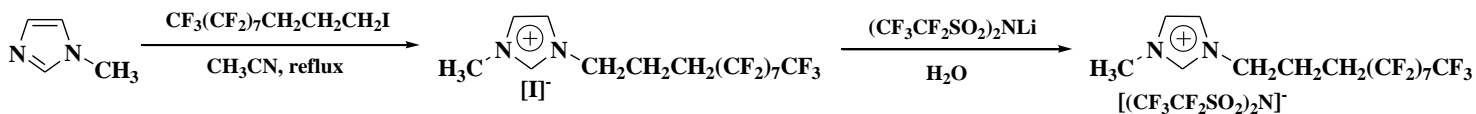

\section{Scheme 1 Synthesis of F-IL.}

Typically, $0.7 \mathrm{~g}(8.5 \mathrm{mmol})$ of 1-methylimidazole and $5.0 \mathrm{~g}(8.5 \mathrm{mmol})$ of 3 (perfluorooctyl)propyl iodide were dissolved into $20 \mathrm{~mL}$ of acetonitrile. The mixture was refluxed at $90{ }^{\circ} \mathrm{C}$ for $24 \mathrm{~h}$, and subsequently cooled down to room temperature. The resulting samples were filtered and washed with ethyl ether for 3 times. The 1-(3perfluorooctyl)propyl-3-methylimidazolium iodide (Iodide-IL) was obtained as a white powder after being dried in a vacuum oven. The yield from this reaction was $95.6 \%$. The spectra of ${ }^{1} \mathrm{H}$ NMR (DMSO- $d_{6}$ as the solvent, $\delta$, ppm) were $9.083(\mathrm{~N}-\boldsymbol{C H}-\mathrm{N}, \mathrm{s}, 1 \mathrm{H}), 7.779$ $(\mathrm{N}-\boldsymbol{C H}-\mathrm{CH}, \mathrm{s}, 1 \mathrm{H}), 7.698(\mathrm{~N}-\boldsymbol{C H}-\mathrm{CH}, \mathrm{s}, 1 \mathrm{H}), 4.261\left(\mathrm{~N}-\boldsymbol{C H}_{2}, \mathrm{t}, 2 \mathrm{H}\right), 3.834\left(\mathrm{~N}-\boldsymbol{C H}_{3}, \mathrm{~s}, 3 \mathrm{H}\right)$, $2.320\left(\mathrm{~N}-\mathrm{CH}_{2}-\boldsymbol{C H}_{2}, \mathrm{~m}, 2 \mathrm{H}\right), 2.089\left(\mathrm{~N}-\mathrm{CH}_{2}-\mathrm{CH}_{2}-\boldsymbol{C H}_{2}-\left(\mathrm{CF}_{2}\right)_{7} \mathrm{CF}_{3}, \mathrm{~m}, 2 \mathrm{H}\right)$.

$1.5 \mathrm{~g}$ (2.2 mmol) of 1-(3-perfluorooctyl)propyl-3-methylimidazolium iodide was dispersed into $50 \mathrm{~mL}$ of water. $1.0 \mathrm{~g}(2.5 \mathrm{mmol})$ of bis(perfluoroethylsulfonyl)amide lithium salt was slowly added into this suspension under vigorous stirring. The mixture was stirred at room temperature for $24 \mathrm{~h}$ and was filtered to remove water. After washing with water for 3 times, the white solid F-IL was dried in a vacuum oven. The yield of this product was $95.0 \%$. 
The spectra of ${ }^{1} \mathrm{H}$ NMR (DMSO- $d_{6}$ as the solvent, $\delta$, ppm) were $9.073(\mathrm{~N}-\boldsymbol{C H}-\mathrm{N}, \mathrm{s}, 1 \mathrm{H})$, $7.772(\mathrm{~N}-\boldsymbol{C H}-\mathrm{CH}, \mathrm{s}, 1 \mathrm{H}), 7.691(\mathrm{~N}-\boldsymbol{C H}-\mathrm{CH}, \mathrm{s}, 1 \mathrm{H}), 4.255\left(\mathrm{~N}-\boldsymbol{C H}_{2}, \mathrm{t}, 2 \mathrm{H}\right), 3.830\left(\mathrm{~N}-\boldsymbol{C H}_{3}, \mathrm{~s}\right.$, $3 \mathrm{H}), 2.288\left(\mathrm{~N}-\mathrm{CH}_{2}-\boldsymbol{C H}_{2}, \mathrm{~m}, 2 \mathrm{H}\right), 2.076\left(\mathrm{~N}-\mathrm{CH}_{2}-\mathrm{CH}_{2}-\boldsymbol{C H}_{2}-\left(\mathrm{CF}_{2}\right)_{7} \mathrm{CF}_{3}, \mathrm{~m}, 2 \mathrm{H}\right)$.

\subsection{Oxidation of MWCNTs}

The oxidized MWCNTs were prepared with the mixture of concentrated nitrite acid and sulfuric acid (the volumetric ratio is: $\mathrm{H}_{2} \mathrm{SO}_{4} / \mathrm{HNO}_{3}=3: 1$ ). In details, $2 \mathrm{~g}$ of MWCNTs was dispersed in the acid mixture and sonicated at $35{ }^{\circ} \mathrm{C}$ for $5 \mathrm{~h}$ followed by stirred at $45{ }^{\circ} \mathrm{C}$ for $24 \mathrm{~h}$. The final product was filtrated with $0.1 \mu \mathrm{m}$-VVLP membrane (Millipore) and washed with water, tetrahydrofuran (THF), and ethanol consequently until $\mathrm{pH}$ was neutral. After drying in vacuum oven, the carboxylate groups of the oxidized MWCNTs determined by titration experiment was $2.0 \mathrm{mmol} / \mathrm{g}$ [23].

\subsection{Modification of MWCNTs with F-IL}

$0.05 \mathrm{~g}$ of oxidized MWCNTs and $0.05 \mathrm{~g}(0.07 \mathrm{mmol})$ of 1-(3-perfluorooctyl)propyl3-methylimidazolium iodide (Iodide-IL) were dispersed in $100 \mathrm{~mL}$ of DI water by sonication of the suspensions over night. $0.03 \mathrm{~g}(0.07 \mathrm{mmol})$ of bis(perfluoroethylsulfonyl)amide lithium salt (dissolved in $10 \mathrm{~mL}$ DI water) was slowly added into this suspension under vigorous stirring. The in-situ produced F-IL modified MWCNTs were precipitated gradually and floated on the solution. The F-IL-MWCNTs powder were then collected by filtration of the suspension and dried in a vacuum oven for $24 \mathrm{~h}$.

\subsection{Preparation of FEP Nanocomposite Films}


Pristine MWCNTs and F-IL-MWCNTs were mixed with FEP pellets using a twinscrew blender $(100 \mathrm{rpm})$ at $290{ }^{\circ} \mathrm{C}$ for $10 \mathrm{~min}$. All samples were melt-pressed at $282{ }^{\circ} \mathrm{C}$ into films with the thickness of around $0.10 \mathrm{~mm}$. The composition of the nanocomposites was listed in Table $1[7,15,24]$.

Table 1 Composition of FEP nanocomposites

\begin{tabular}{ll}
\hline Nanocomposites & Composition (weight ratio) \\
\hline F-IL-MWCNTs & F-IL $:$ MWCNTs $=1: 1$ \\
N1 & FEP $:$ MWCNTs $=10: 1$ \\
N2 & FEP $:$ f-IL-MWCNTs $=10: 1$ \\
\hline
\end{tabular}

\subsection{Measurements}

Thermal gravimetric analysis (TGA) scans of the samples were collected at $10{ }^{\circ} \mathrm{C} / \mathrm{min}$ from $60{ }^{\circ} \mathrm{C}$ to $800{ }^{\circ} \mathrm{C}$ under an air flow using the TGA7 instrument from Perkin-Elmer Inc.. Differential scanning calorimeter (DSC) scans of the samples were collected at $10{ }^{\circ} \mathrm{C} / \mathrm{min}$ from $25{ }^{\circ} \mathrm{C}$ to $350{ }^{\circ} \mathrm{C}$ under a nitrogen flow (20 mL/min) using the DSC-7 instrument from Perkin Elmer, Inc. with the mechanically refrigerated cooling system (FTS Systems, Inc.). A scanning electron microscope (SEM, LEO 1550) equipped with a Schottky field emission gun $(10 \mathrm{kV})$ and a Robinson backscatter detector was used for the SEM micrographs in crosssectional views. The cross-sectioned samples were prepared by freeze-fracturing of the film in a liquid nitrogen bath and sputtered with gold in an argon atmosphere before observation. The ${ }^{1} \mathrm{H}$ NMR (nuclear magnetic resonance) spectrum was taken at $25{ }^{\circ} \mathrm{C}$ on a Varian GEMINI 2300 NMR spectrometer with DMSO- $d_{6}$ as the solvent. All melt-pressed film samples were uniaxially stretched at room temperature using a modified Instron 4442 tensile apparatus, where symmetric deformation was carried out. The initial length between the Instron jaws was $10 \mathrm{~mm}$ and the chosen stretching rate was $5 \mathrm{~mm} / \mathrm{min}$. Each type of sample was repeated for 3 times and average results were plotted. All data were collected 
Simultaneous wide-angle X-ray diffraction (WAXD) and small-angle X-ray scattering (SAXS) experiments were carried out at beamline X27C in the National Synchrotron Light Source (NSLS), Brookhaven National Laboratory (BNL). The wavelength used was 0.1371 $\mathrm{nm}$. The sample-to-detector distance for WAXD was $108.8 \mathrm{~mm}$, and that for SAXS was $1883.3 \mathrm{~mm}$. A two-dimensional MAR-CCD (MAR, Inc.) X-ray detector was used for the real-time data collection during sample stretching. A typical image acquisition time was $20 \mathrm{~s}$ per image.

\section{Results and Discussion}

\subsection{Thermal Stabilities of Nanofillers}

As well-known that FEP has to be processed at high temperature above its melting point (i.e., $257.7^{\circ} \mathrm{C}$ ), thus, all nanofillers introduced to improve the processability or mechanical properties of FEP have been required to exhibit high temperature tolerance. The onset decomposition temperatures of the synthesized fluorinated ionic liquids as well as FEP were determined with TGA, as shown in Figure 1 (A).

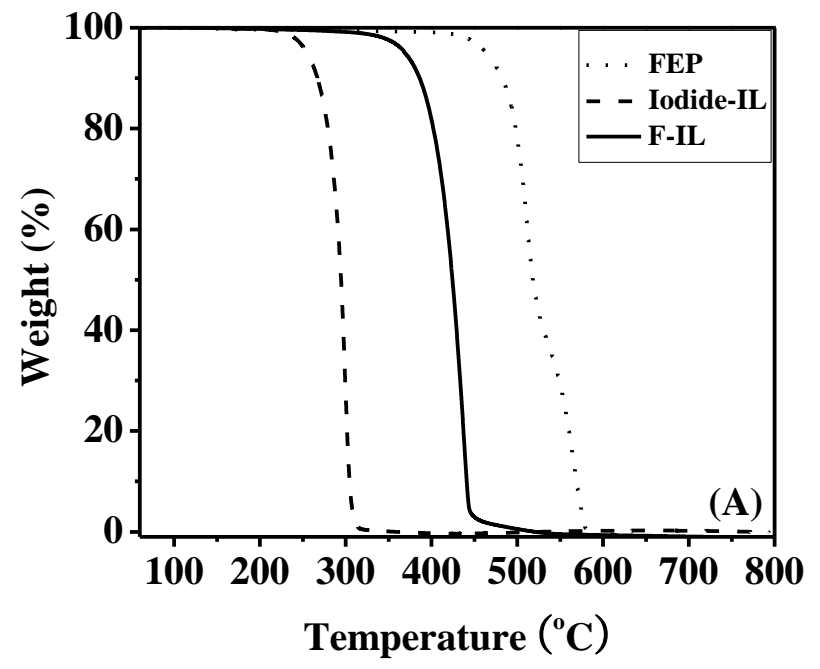




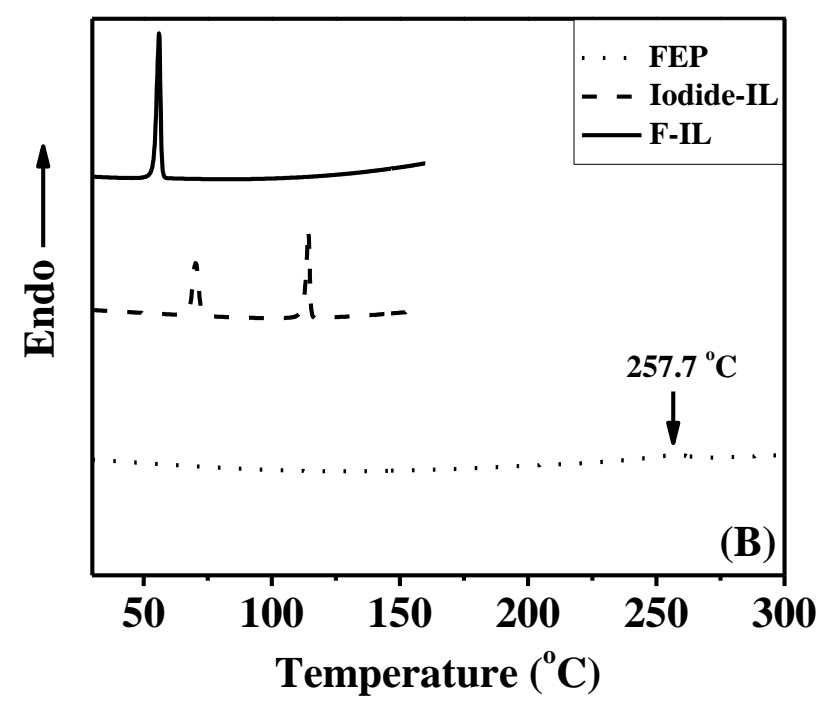

Figure 1 Thermal stabilities (A) and melting points (B) of FEP and synthesized ionic liquids.

The onset decomposition temperature of FEP was $464.3{ }^{\circ} \mathrm{C}(5 \%$ weight loss $)$ at air atmosphere indicated that the processes of fluorocopolymer FEP have to be conducted under this threshold temperature. It was interesting to note that fluorinated ILs with different anions exhibit different thermal stabilities. The decomposition temperature of fluorinated ILs essentially relay on the type of anions, where iodide-IL has much lower decomposition temperature of $255.2{ }^{\circ} \mathrm{C}$ than that of bis(perfluoroethylsufonyl)amine-IL (F-IL, 367.0 ${ }^{\circ} \mathrm{C}$ ). Therefore, iodide-IL could not be used safely at the temperature higher than $250{ }^{\circ} \mathrm{C}$, while FIL can be applied in the production process required high-temperature as $360{ }^{\circ} \mathrm{C}$ without obvious decomposition. Furthermore, FEP nanocomposite can only be fabricated by extrusion at above $257.7{ }^{\circ} \mathrm{C}$ (the melting point of FEP), indicating that the practical temperature for extrusion of FEP or its nanocomposite would be $290{ }^{\circ} \mathrm{C}$ (about $30{ }^{\circ} \mathrm{C}$ higher than the melting points of FEP). At that temperature, iodine-IL could no longer be qualified, while F-IL can be employed without concern of decomposition (Figure 1 (A)). Moreover, the melting point of F-IL was $56.0{ }^{\circ} \mathrm{C}$, which is liquid state (low viscosity) at the extrusion 
temperature and consequently, would be benefit to decrease the extruding viscosity of the FEP nanocomposite.

Due to the high decomposition temperature of F-IL-MWCNTs of $370.3{ }^{\circ} \mathrm{C}$, the FEP nanocomposite incorporated with F-IL-MWCNTs exhibits high thermal stability similar to that of pure FEP, as shown in Figure 2 (A). Therefore, at the extrusion temperature, i.e., 290 ${ }^{\circ} \mathrm{C}$, the nanocomposite with nanofillers could be processed safely without thermal stability concern as expected.
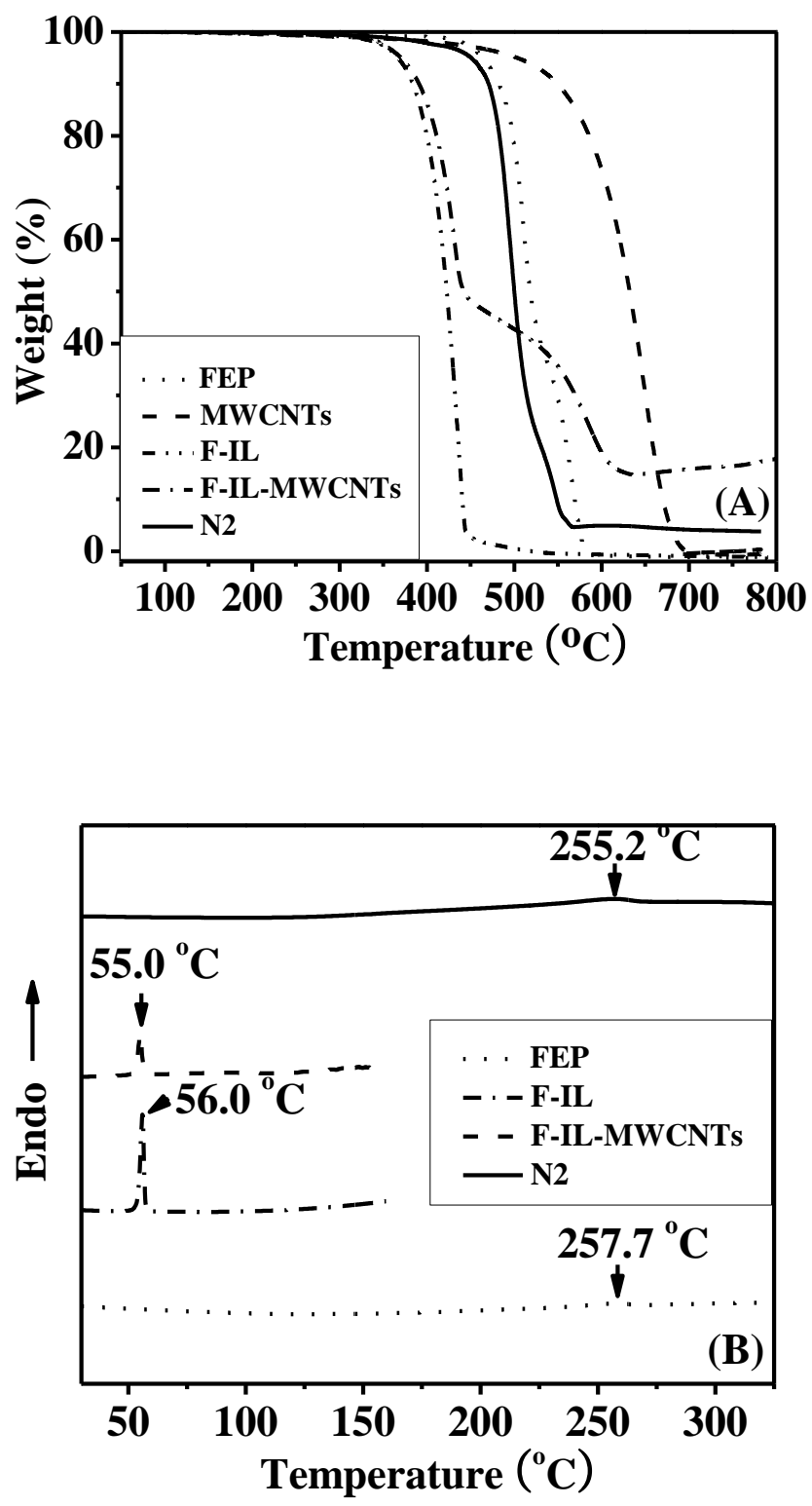
Figure 2 Thermal stabilities (A) and melting points (B) of F-IL-MWCNTs and FEP nanocomposite.

From Figure 2 (A), it can be seen that nanocomposite N2 was decomposed at 450.8 which were comparable to $464.3{ }^{\circ} \mathrm{C}$ of pure FEP after incorporated F-IL-MWCNTs by extruding at $290{ }^{\circ} \mathrm{C}$. Further determination of the melting point of $\mathrm{N} 2$ was carried out to estimate the dispersity of nanofillers, as shown in Figure 2 (B). It was found that the nanocomposite $\mathrm{N} 2$ has a unique melting point indicating the well-dispersity of additive F-ILMWCNTs, which was decreased about $2.5{ }^{\circ} \mathrm{C}$ when compared to pure FEP. Moreover, the melting point of F-IL-MWCNTs was $1{ }^{\circ} \mathrm{C}$-lower than that of pure F-IL, which could be regarded as an evidence of the interaction between F-IL and MWCNTs.

\subsection{Surface Morphologies of MWCNTs, FEP, and N2}

To determine the dispersity of F-IL modified MWCNTs, the morphologies of MWCNTs, FEP, and N2 could be observed directly by SEM, as shown in Figure 3.
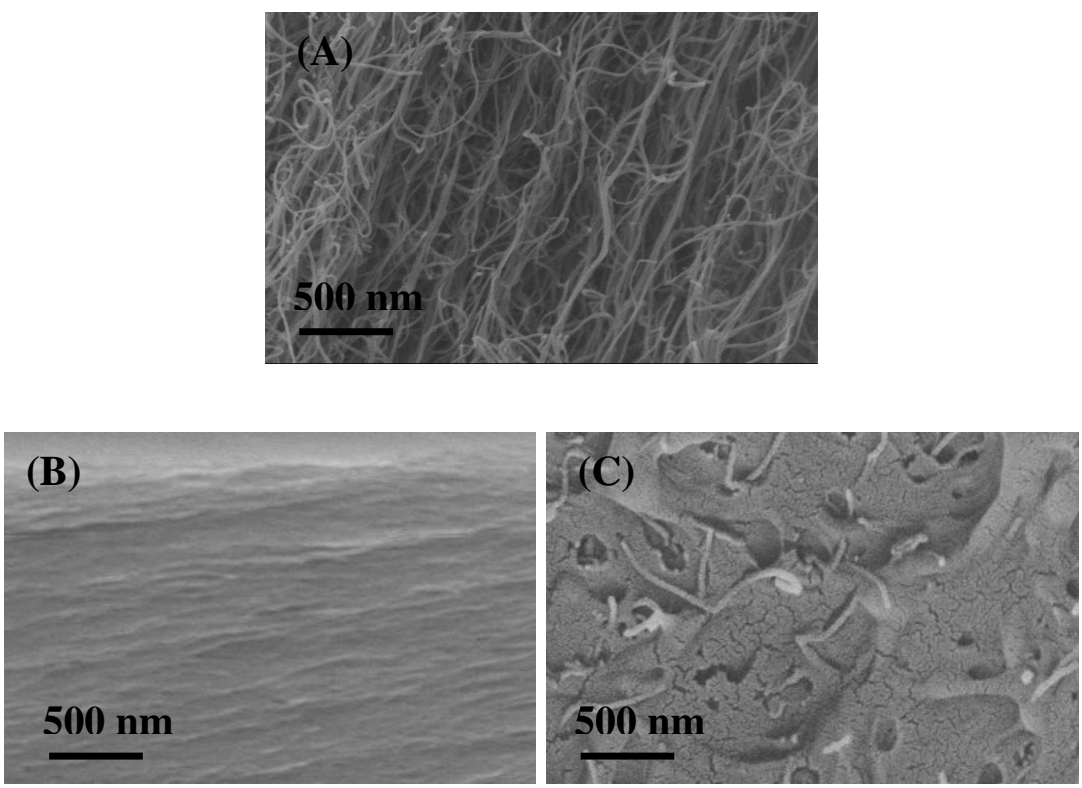
Figure 3 SEM images of MWCNTs (A), cross-sectional views of FEP (B) and the nanocomposite $\mathrm{N} 2(\mathrm{C})$.

Usually, MWCNTs are entangled each other and are very different to be dispersed individually in a polymer matrix, e.g., FEP (Figure 3 (B)). However, the modified MWCNTs with fluorinated ionic liquid exhibited good compatibility with FEP due to the surface of MWCNTs covered with fluorinated substituted alkyl groups of the fluorinated IL. As can be seen in Figure $3(\mathrm{C})$, the F-IL-MWCNTs were dispersed individually in FEP, which was expected to improve mechanical properties and processability of pure FEP.

\subsection{Interaction of Fluorinated Ionic Liquid with MWCNTs}

To evaluate the interaction of F-IL with MWCNTs, 1 D WAXD intensity profiles were shown in Figure 4.

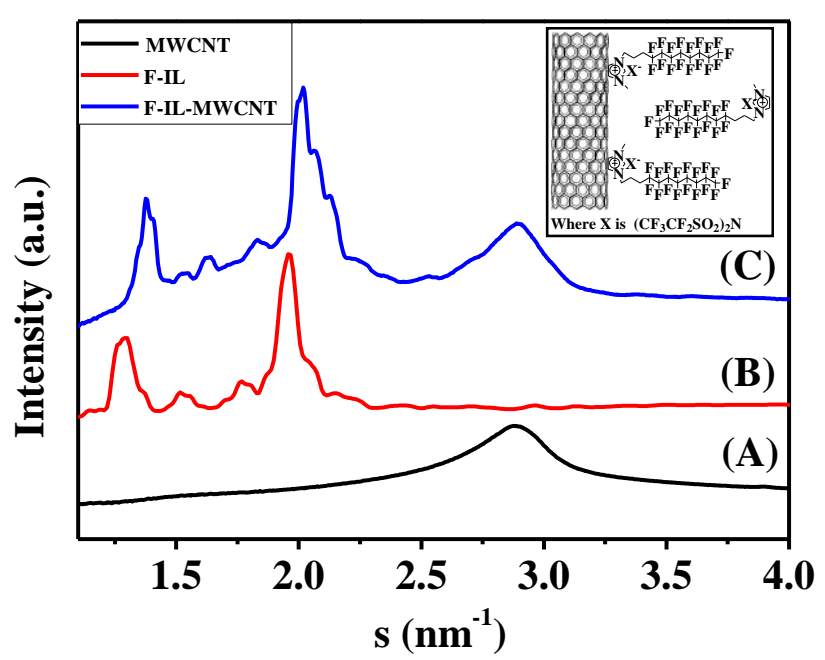

Figure 4 1D WAXD intensity profiles of MWCNTs (A), F-IL, and F-IL-MWCNTs (C). The insert picture is a schematic representation of the $\pi$-stacking arrangement of F-IL on a MWCNT surface. 
The 1-D WAXD intensity profile of F-IL (Figure 4 (A)) show 4 peaks at $s$ equal to 1.30, $1.52,1.77$ and $1.96 \mathrm{~nm}^{-1}$, which moved to $1.38,1.58,1.83$, and $2.02 \mathrm{~nm}^{-1}$ in the diffraction profile of F-IL-MWCNT, respectively, due to the interaction between F-IL and MWCNTs evidenced by increasing the d-spacing of diffraction peaks. The profile of MWCNTs has only one diffraction peak at $\mathrm{s}=2.89 \mathrm{~nm}^{-1}$. There is a new peak appeared at $\mathrm{s}=2.07 \mathrm{~nm}^{-1}$ which corresponds to $\mathrm{d}$-spacing of $0.48 \mathrm{~nm}$ because of a $\pi$-stacking arrangement of the imidazolium cationic head on MWCNTs surface [25].

\subsection{Mechanical Properties of Nanocomposites N1 and N2}

The mechanical properties of nanocomposites N1 and N2 were determined by tensile tests at room temperature with pure FEP as the control sample, as shown in Figure 5.

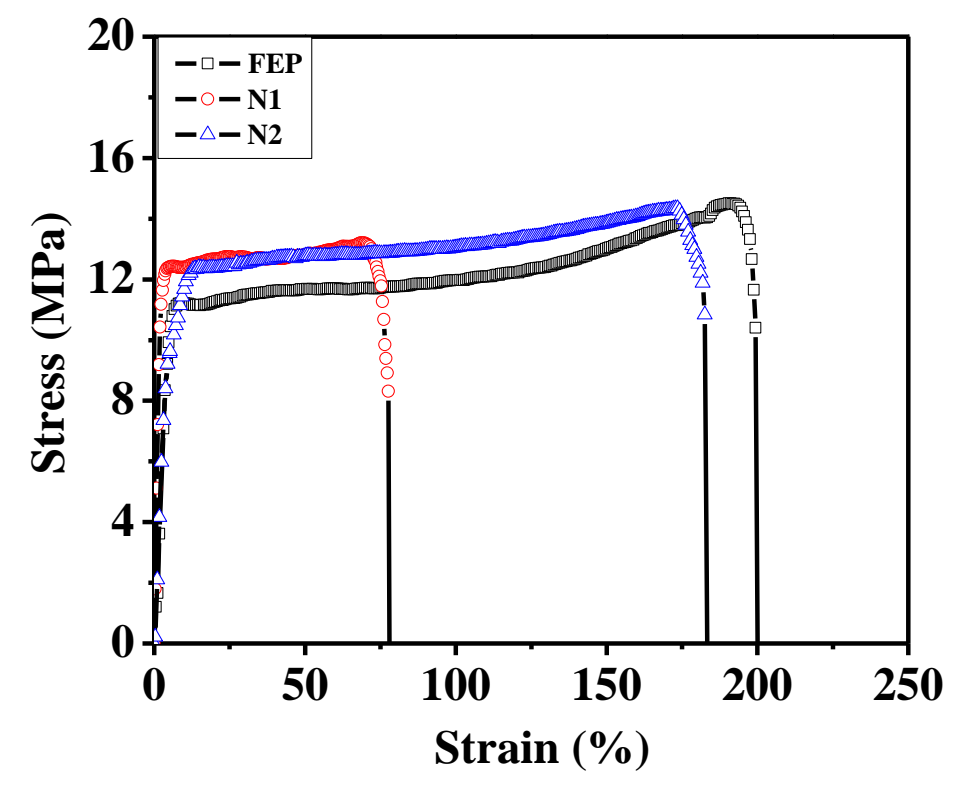

Figure 5 Mechanical properties of nanocomposites N1 and N2.

A good dispersion of MWCNTs in FEP has been verified through tensile experiments when MWCNTs with and without F-IL modification were involved. It was seen that pristine MWCNTs incorporated in FEP matrix (N1) can increase the stretching modulus compared to 
that of pure FEP, however, elongation to break ratio of the nanocomposite N1 decreased essentially due to the poor compatibility of MWCNTs in FEP. To improve the compatibility, MWCNTs have been modified with F-IL through forming "cation- $\pi$ " complex, while the elongation to break ratio of $\mathrm{N} 2$ was much higher than that of $\mathrm{N} 1$ which could be attributed to the better plasticization effect of F-IL-MWCNT in the FEP matrix. Meanwhile, the stretching modulus of $\mathrm{N} 2$ remains the same as $\mathrm{N} 1$ and higher than that of pure FEP. Therefore, nanofillers F-IL-MWCNTs play two possible roles in FEP processing as (1) plasticizer to improve the processability of FEP; and (2) nanofillers to improve the dispersity of MWCNTs in FEP, and therefore, to enhance the mechanical properties of FEP by a synergistic effect of the fluorinated ionic liquid and MWCNTs.

\subsection{WAXD and SAXS Study on Nanocomposite during Tensile Deformation}

Three diffraction peaks of (100), (110), and (200) could be observed in the 2-D diffraction patterns which were attributed to the typical crystal structure of FEP [7, 26, 27], where peak (002) was assigned as the diffraction of MWCNTs with $\mathrm{s}=2.95 \mathrm{~nm}^{-1}$, as shown in Figure 6 (A). From the WAXD profiles, the (100), (110), and (200) diffraction intensity became aggregated around the equator with increasing strain. The SAXS patterns of N2 clearly showed that F-IL-MWCNTs greatly affected the FEP morphology. The equatorial streak evolved immediately upon stretching and became sharper with increasing strain, as shown in Figure $6(\mathrm{~B})$. 

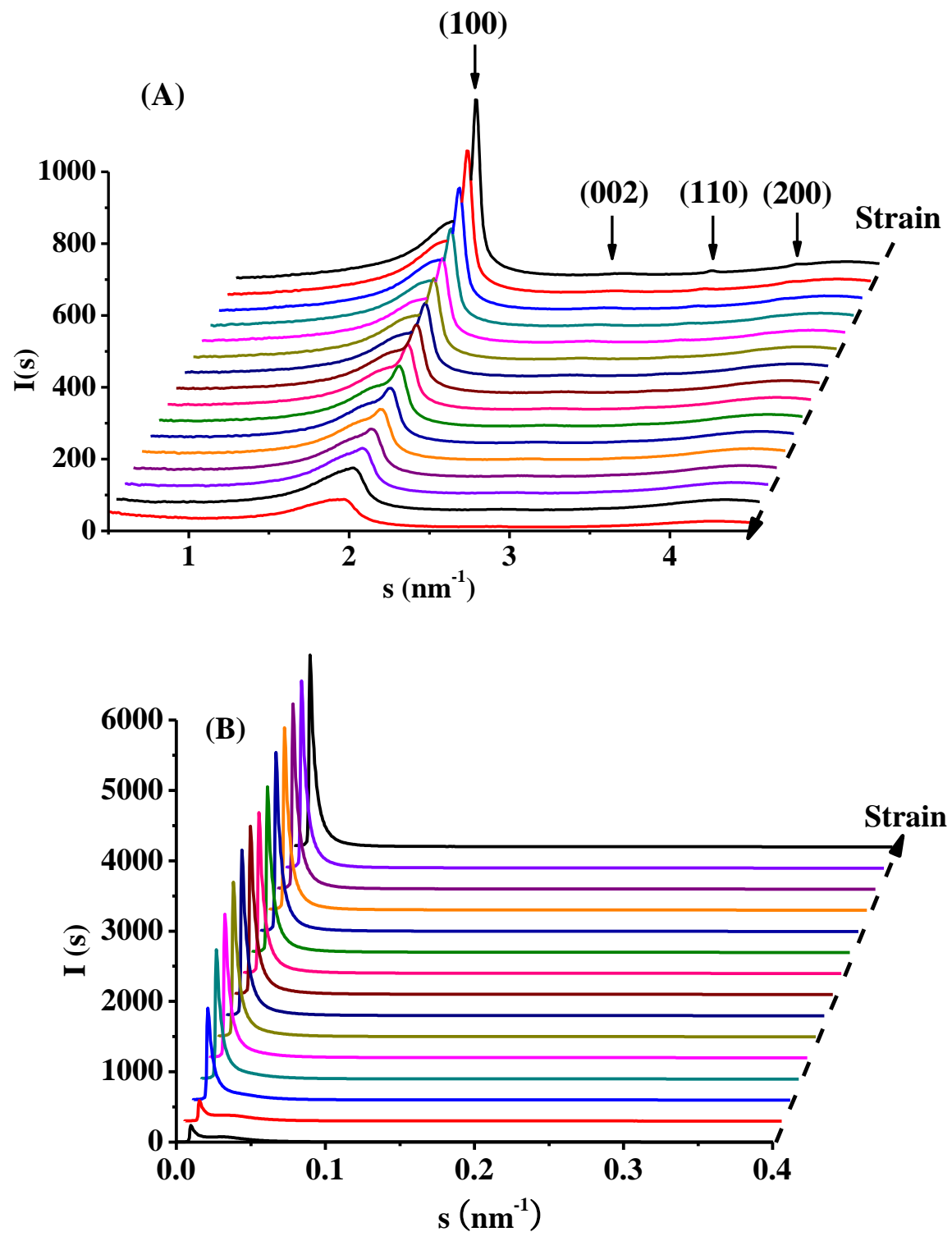

Figure 6 WAXD (A) and SAXS (B) profiles of nanocomposite N2 at varying strains during the tensile deformation.

It is conceivable that at sufficiently high nanofiller concentrations the interactions of nano-sized additives would generate a rigid network structure (a scaffold), which not only can carry a significant fraction of stress but also would disrupt the morphology of the matrix, even though, the total crystallinity of FEP matrix appeared to be unchanged in the presence of F-IL-MWCNTs. 


\section{Conclusions}

The improving processability and mechanical properties of fluorocopolymer, i.e. FEP, has been carried out with novel fluorinated ionic liquid-modified MWCNTs and verified with SEM, tensile tests, and synchrotron WAXD and SAXS. The fluorinated ionic liquid has high thermal stability and lower melting point issued by the anion of bis(perfluoroethylsufonyl)amine, which is benefit to change the processability of FEP. MWCNTs modified with the fluorinated ionic liquid (F-IL-MWCNTs) can be dispersed well in FEP matrix and their corresponding nanocomposite $\mathrm{N} 2$ exhibits higher mechanical properties than that of pure FEP. To be specific, F-IL-MWCNTs served as both of plasticizer and reinforcing reagent in FEP nanocomposite during the stretching deformation evidenced by tensile tests and WAXD/SAXS measurements.

\section{Acknowledgements}

The financial support for this work was provided by a grant from the SusChEM Program of the National Science Foundation (DMR-1409507). H.M. would like to acknowledge the Research Fund from the State Key Laboratory of Organic-Inorganic Composites at Beijing University of Chemical Technology (oic-201503004) and the Fundamental Research Funds for the Central Universities (buctrc201501) for partial support of this work.

\section{References}

1. Tervoort, T.; Visjager, J.; Graf, B.; Smith, P. Melt-Processable Poly(tetrafluoroethylene). Macromolecules 2000, 33, 6460-6465.

2. Akinci, A.; Cobanoglu, E. Coating of Al mould surfaces with polytetrafluoroethylene (PTFE), fluorinated ethylene propylene (FEP), perfluoro-alkoxy (PFA) and ethylene- 
tetrafluoroethylene (ETFE). E-Polymer 2009, no033, 1-7.

3. Patri, M.; Hande, V. R.; Phadnis, S.; Deb, P. C. Radiation-grafted solid polymer electrolyte membrane: studies of fluorinated ethylene propylene (FEP) copolymer-gacrylic acid grafted membranes and their sulfonated derivatives. Polymers for Advanced Technologies 2004, 15, 485-489.

4. Ajayan, P. M. Nanotubes from carbon. Chemical Reviews 1999, 99, 1787-1799.

5. Ho, K. K. C.; Kalinka, G.; Tran, M. Q.; Polyakova, N. V.; Bismarck, A. Fluorinated carbon fibres and their suitability as reinforcement for fluoropolymers. Composites Science and Technology 2007, 67, 2699-2706.

6. Hsiao, B. S.; Chu, B.; Wei, J.; Ma, H. Y.; Zuo, F. Ionic liquids, functionalized particulates, and fluoropolymer composites. US patent No. US8563657.

7. Chen, X.; Burger, C.; Fang, D.; Sics, I; Wang, X.; He, W; Somani, R. H.; Yoon, K.; Hsiao, B.S.; Chu, B. In-situ X-ray deformation study of fluorinated multiwalled carbon nanotube and fluorinated ethylene-propylene nanocomposite fibers. Macromolecules 2006, 39, 5427-5437.

8. Tasis, D.; Tagmatarchis, N; Bianco, A.; Prato, M. Chemistry of Carbon Nanotubes. Chemical Reviews 2006, 106, 1105-1136.

9. Eder, D. Carbon Nanotube-Inorganic Hybrids. Chemical Reviews 2010, 110, 13481385.

10. Fukushima, T.; Kosaka, A,; Ishimura. Y.; Yamamoto, T.; Takigawa, T.; Ishii, N.; Aida, T. Molecular ordering of organic molten salts triggered by single-walled carbon nanotubes. Science 2003, 300, 2072-2074.

11. Fukushima, T. $\pi$-Electronic soft materials based on graphitic nanostructures. Polymer Journal 2006, 38, 743-756.

12. Tunckol, M.; Durand, J.; Serp, P. Carbon nanomaterial-ionic liquid hybrids. Carbon 
2012, 50, 4303-4334.

13. Ding, Y.; Su, D. S. Host-guest nanocomposites of multiwalled carbon nanotubes and ionic liquids with controllable composition. ChemSusChem 2014, 7, 1542-1546.

14. Bellayer, S.; Gilman, J. W.; Eidelman, N.; Bourbigot, S.; Flambard, X.; Fox, D. M.; De Long, H. C.; Trulove, P. C. Preparation of homogeneously dispersed multiwalled carbon nanotube/polystyrene nanocomposites via melt extrusion using trialkyl imidazolium compatibilizer. Advanced Functional Materials 2005, 15, 910-916.

15. Ma, H. Y.; Chen, X.; Hsiao, B. S.; Chu, B. Improving toughness of ultra-high molecular weight polyethylene with ionic liquid modified carbon nanofiber, Polymer $2014,55,160-165$.

16. Li, X.; Mao, Y.; Ma, H. Y.; Zuo, F.; Hsiao, B. S.; Chu, B. An in-situ X-ray scattering study during uniaxial stretching of ionic liquid/ultra-high molecular weight polyethylene blends. Polymer 2011, 52, 4610-4618.

17. Zhao, L.; Li, Y.; Cao, X.; You, J.; Dong, W. Multifunctional role of an ionic liquid in melt-blended poly(methyl methacrylate)/multi-walled carbon nanotube nanocomposites. Nanotechnology 2012, 23, 255702.

18. Subramaniam, K.; Das, A.; Haubler, L.; Harnisch, C.; Stockelhuber, K. W.; Heinrich, G. Enhanced thermal stability of polychloroprene rubber composites with ionic liquid modified MWCNTs. Polymer Degradation and Stability 2012, 97, 776-785.

19. Livi, S.; Duchet-Rumeau, J.; Pham, T. N.; Gerard, J-F. Synthesis and physical properties of new surfactants based on ionic liquids: Improvement of thermal stability and mechanical behaviour of high density polyethylene nanocomposites. Journal of Colloid and Interface Science 2011, 354, 555-562.

20. Zeng, Z.; Phillips, B. S.; Xiao, J. C.; Shreeve, J. M. Polyfluoroalkyl, polyethylene glycol, 1,4-bismethylenebenzene, or 1,4-bismethylene-2,3,5,6-tetrafluorobenzene 
bridged functionalized dicationic ionic liquids: synthesis and properties as high temperature lubricants. Chemistry of Materials 2008, 20, 2719-2726.

21. Kim, D. S.; Natalia, D.; Nguyen, D. Q.; Suh, D. J.; Kim, H.; Kim, H. S.; Lee, H. Facile one-pot synthesis of 1-alkyl-3-polyfluoroalkyl imidazolium ionic liquids. Synlett 2009, 13, 2101-2104.

22. Bara, J. E.; Gabriel, C. J.; Carlisle, T. K.; Camper, D. E.; Finotello, A.; Gin, D L.; Noble, R. D. Gas separations in fluoroalkyl-functionalized room-temperature ionic liquids using supported liquid membranes. Chemical Engineering Journal 2009, 147, 43-50.

23. Gonzalez-Guerrero, A. B.; Mendoza, E.; Pellicer, E.; Alsina, F.; Fernandez-Sanchez, C.; Lechuga, L. M. Discriminating the carboxylic groups from the total acidic sites in oxidized multi-wall carbon nanotubes by means of acid-base titration. Chemical Physics Letters 2008, 462, 256-259.

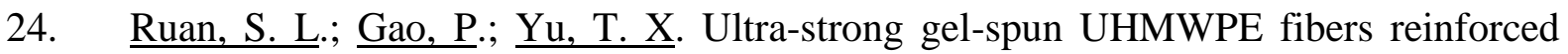
using multiwalled carbon nanotubes. Polymer 2006, 47, 1604-1611.

25. Ma, J. C.; Dougherty, D. A. The cation- $\pi$ interaction. Chemical Reviews 1997, 97, 1303-1324.

26. White, M. L.; Waddon, A. J.; Atkins, E. D. T.; Farris, R. J. Copolymerization of hexafluoropropylene and tetrafluoroethylene: Effect on chain conformation and crystal structure. Journal of Polymer Science Part B: Polymer Physics 1998, 36, 28112819.

27. Endo, M.; Ohnishi, A.; Kutsumizu, S.; Shimizu, T.; Yano, S. Crystallization in binary blends of polytetrafluoroethylene with perfluorinated tetrafluoroethylene copolymer. Polymer Journal 2004, 36, 716-727. 


\title{
Modification of Carbon Nanotubes with Fluorinated Ionic Liquid for Improving Processability of Fluoro-Ethylene-Propylene
}

\author{
Hongyang $\mathrm{Ma}^{1,2, *}$, Benjamin $\mathrm{Chu}^{1}$, and Benjamin S. Hsiao ${ }^{1, *}$ \\ ${ }^{1}$ Department of Chemistry, Stony Brook University, Stony Brook, NY 11794, USA \\ ${ }^{2}$ State Key Laboratory of Organic-Inorganic Composites, Beijing University of Chemical \\ Technology, Beijing 100029, China
}

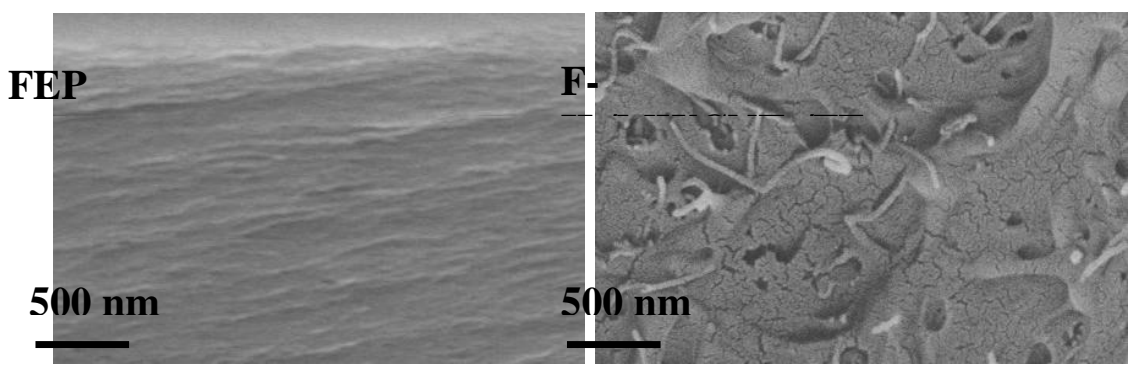

Corresponding authors:

Phone: (631)229-6899 (H.M.); (631)632-7793 (B.S.H). Fax: (631)632-6518

E-mails: mahy@ mail.buct.edu.cn (H.M.); benjamin.hsiao@ stonybrook.edu (B.S.H.) 